Original Research Paper

\title{
Android Based Tourism Guide for Benishangul-Gumuz Region, Ethiopia
}

\author{
Abeselom Befekadu Wolde and Yimer Amedie Muhie \\ Information Technology, Assosa University, Assosa, Ethiopia
}

\author{
Article history \\ Received: 29-07-2020 \\ Revised: 22-10-2020 \\ Accepted: 23-10-2020 \\ Corresponding Author: \\ Abeselom Befekadu \\ Information Technology, \\ Assosa University, Assosa, \\ Ethiopia \\ Email: abeselom2010@gmail.com
}

\begin{abstract}
Benishangul Gumuz is one of the regions which have a potential of tourism in Ethiopia. In order to visit the region tourists have to go to tour operators office or must have a printed guide booklet of the attraction sites. Due to the lack of updated information, tourists are not be able to visit all tourism attraction sites. This research project tries to provide convenience to the visitors by providing a solution to the problems that the tourists faced during their visit. This paper presents the work in developing tourism guide application to replace the old way of tourism guide. The development is based on android platform and delivered as a mobile application. The main objective of this project is to provide tourists the information that they need before and during visits without missing attractions.
\end{abstract}

Keywords: Tour Guide, Android, Google Map, Smart Devices, Web Server, Offline Tour

\section{Introduction}

Nowadays the lives of people have been made easier because of the significant improvement of smart phones and the applications development during the past few years. This days' smart phones provide extra services in addition to voice communications.

Today tourists want to get information about their destination before departing to the sites. Mobile based tour guide system allows them to get the needed information at any time, from anywhere. The goal of this system is to replace paper-based tour guide with mobile application.

This application is basically about travelling sites in Benishangul Gumuz Region. For pleasure it clearly gives someone a picture of what they should expect on the arrival, it shows the beauty of the region and the attractions.

\section{Problem of Statement}

Because of the lack of technology, tourists have a problem of accessing tourism information in Benishangul Gumuz region. Both national and international tourists have been facing problems in acquiring tourist sites and accommodation during their stay in the region. In order to alleviate this trouble, this research project purposes the development of android based tourism guide application where by the tourist can have information about the places any time anywhere.

\section{Objectives}

The main objective of this research project is to develop android based tourism guide application for Benishangul Gumuz region.

\section{Specific Objectives}

The specific objectives of this research project are:

- To conduct research project on the current tourism sites in Benishangul Gumuz

- To design the android based mobile application including the backend and the front end

- To test the application

- To implement the application

\section{Scope of the Research Project}

The research project has only included recognized tourism sites by Benishangul Gumuz region tourism bureau. This allows the research project to display only legal tourism sites.

\section{Limitations of the Research Project}

Due to the remoteness and lack of infrastructure, the region tourism attractions have not been mapped and 
taken by Google maps. This limits the project from making calculations of distance and time from the sites.

\section{Significance of the Research Project}

This research project is important economically, academically and socially for both to the community and to the government. Below are some of the reasons why this research project is too important:

- Finding tourism sites has been made easy

- The cost of finding information about tourism sites has been cheaper

- For tourists planning of trips to Benishangul Gumuz would have full information

\section{Literature Review}

\section{Mobile Application Development}

At an early stage of software development, requirements collection has been included and the way how to carry out is not different between traditional and mobile application development. Mobile applications have a possibly bigger, more heterogeneous and loosely defined target group of users which has got to be accounted for when doing requirement collection and analysis, but the collection itself can be carried out utilizing the same method in both cases.

The article "Mobile Government User Requirements model" (Al Thunibat et al., 2011) discussed about traditional approach to requirement collection in mobile development. At letter stages of design and implementation of the application the real deference come. In this stage the development tools, programming language used and the frameworks are deferent. Design guidelines for mobile development have been proposed by both (Oinas-Kukkonen and Kurkela, 2003). These guidelines are used to simplify the development of better applications. Oinas-Kukkonen forwards seven key points that are significant for mobile application development:

- Mobility

- Usefulness

- Relevance

- Ease of use

- Fluency of navigation

- User centeredness

- Personalization

These guidelines can be seen as evaluation criteria to the development process and the application itself.

\section{Related Apps}

\section{Citymapper}

Citymapper (2020) provides more information than google, including the alerts of departure and cancelations of the journey in real-time. It operates in around thirty /30/ cities worldwide. It even tells you how long your journey will take by jetpack. The app is also easy to use and playful.

\section{Splittr}

Splittr (2020) gives travelers a simple platform to share costs between friends and others. The app process payment during your travel. What is needed from you is entering the expenses and who paid what as you go. The app also supports multiple currencies. It is also easy to use and perfect for vacation, weekend trips. Finally, the app generates a report on who is next to pay and who owes how much.

\section{Tripit}

Tripit (2020) compiles travel information from your email confirmation for flits, hotels, rental cars, events booking and converts them in to single journey. That you have to do is simply send your emails to the app. This app can also be used for coordinating a group trip.

\section{Sasol eBirds}

The (Sasol eBirds, 2020) of Southern Africa is an application with best interactive features which enhance your birding experience more excited.

"In southern Africa the Sasol eBirds would undoubtedly be my first choice. The text is detailed, the navigation system is user friendly and the opportunity to listen to recordings of calls and songs is a huge asset. Above all, the superb paintings by Norman Arlott and Peter Hayman have stood the test of time and are more detailed, more accurate and more life-like than anything on offer in other birding apps for the region." - Mark Cocker, author of Birds and People.

\section{Design of Android based Tour Guide}

The application has included text, pictures and other guidance information about the site so people can better understand the tourist attractions and can make accurate decisions.

\section{Modules in Application}

- Location of different sites

- Location of hotels

- Culture and values of the region

- Locate it on the map

- Display full information about the sites 


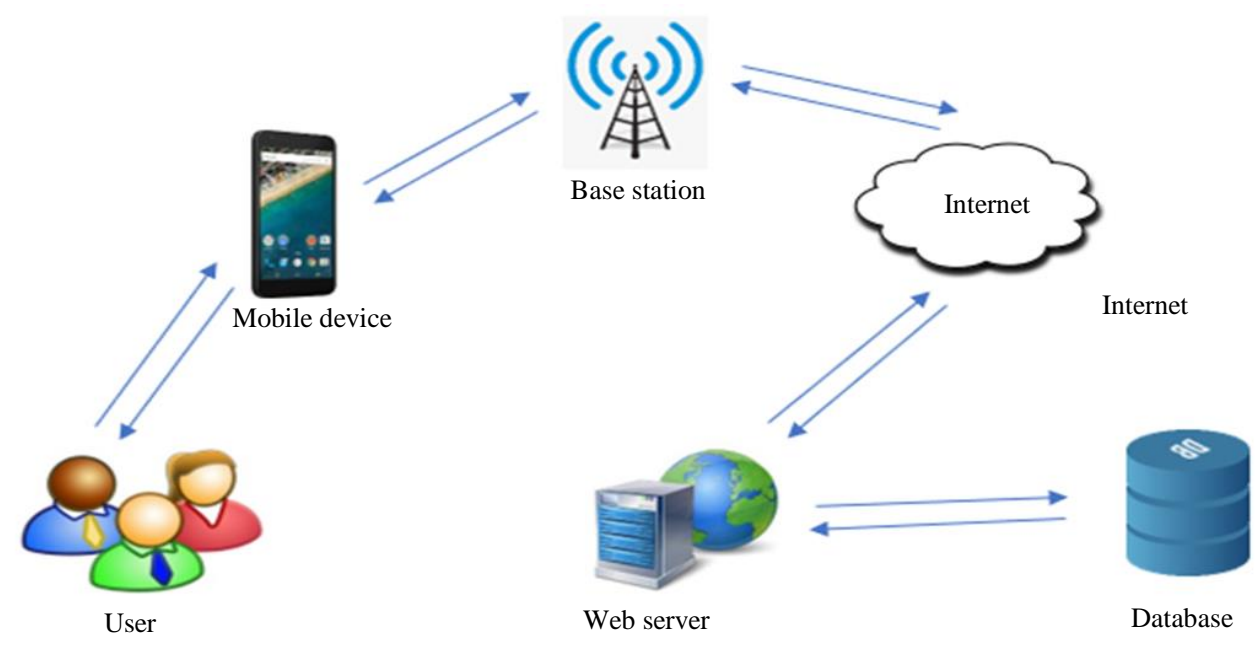

Fig. 1: Architecture of android based tour guide system

The Fig. 1 demonstrates high level architecture of the application. First the user must use android based smart device in order to gain access of the app. Then the web server provides the needed information about the attractions when connected to the network and according to the selection by the user. The mobile app can also be used offline when there is no internet.

\section{Methodology}

This research project is expected to improve the existing long manual way of doing the process. To achieve this, design-oriented research method has been used. Design oriented Research method is basically a problem-solving model (Hevner et al., 2004). The five steps of designoriented method followed in this research project work are problem identification, solution suggestion, development of the application, evaluation and conclusion.

\section{Problem Identification}

The first thing to do in this research project work is to conduct comprehensive review of research projects to acquire a deeper understanding of the research project area and its problem domain. This includes visiting of the tour sites. Existing works related to this research project has been assessed to identify and point out direction in providing solution to the problems.

\section{Solution Suggestion}

The second phase of this research project is to determine different solutions for the identified problems. The suggested solution for the problems was developing android based tour guide mobile application.

\section{Development of the Research Project}

By putting photos of the sites with the link of information on tour guide application, the users can get information they need at any time anywhere so the process of developing the tour guide mobile application has incorporated this service in order to get the exact information of the tour sites.

\section{Evaluation}

Finally, the tour guide application has been evaluated by using different software testing mechanisms such as user, system and unit testing.

\section{Conclusion}

The results found during evaluation phase and the achievements of the tour guide mobile application has been discussed and summarized.

\section{Tools}

In order to conduct the whole research project, there are tools which has been used. These are listed below:

\section{Software Tools}

- Android studio

- SQL lite

- Adobe photoshop

- Draw max

- Google map

- Apache web server

\section{Hard Ware Tools}

- Computer

- Android based mobile device

- $\quad$ Camera (HD)

\section{Implementation and Result}

The application is designed and implemented by using Android Studio and the Android API 29 (Android 9) has been used as the target system for the development of the application. 


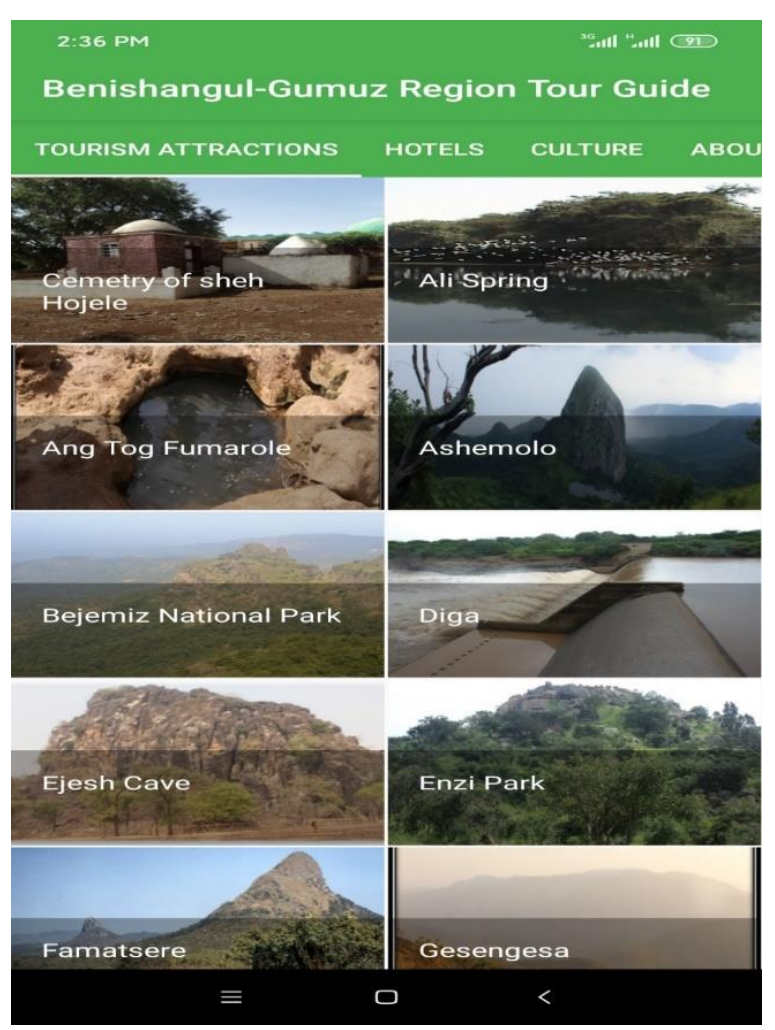

Fig. 2: Attractions part of the app

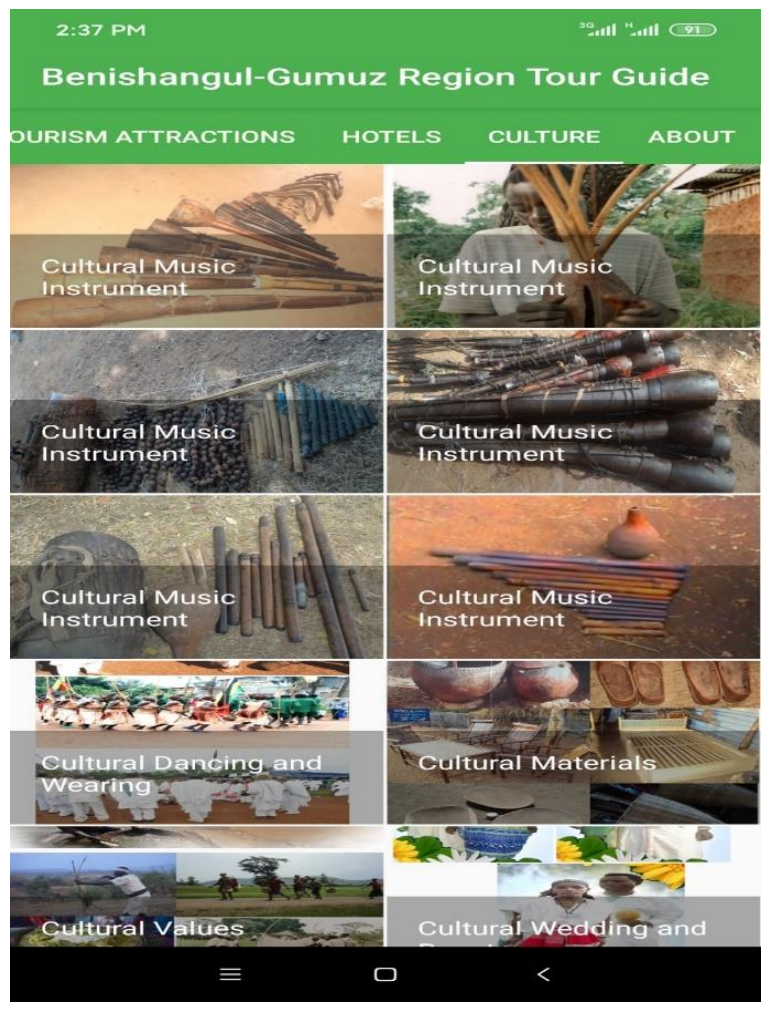

Fig. 3: Culture part of the app

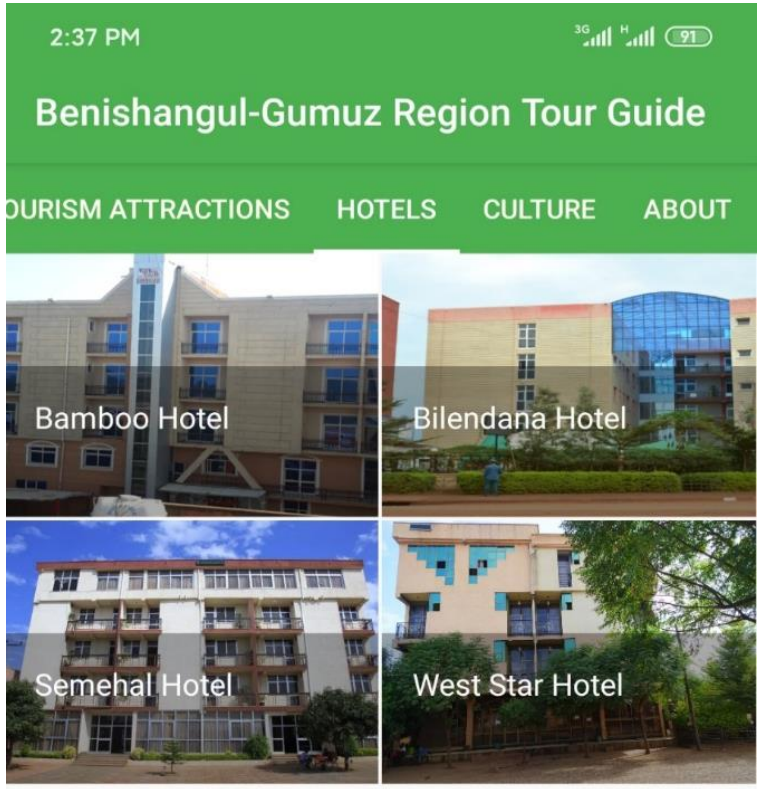

Fig. 4: Hotels part of the app

The main idea behind this research project is to alleviate the problem around identification and promotion of tourism sites in Benishangul Gumuz regional state of Ethiopia. After successfully developing prototype of the application, the interfaces of the prototype look like the Fig. 2-4.

\section{Conclusion}

For so long and still tourism attraction sites and assets in Benishangul Gumuz region have not been promoted enough. The reason behind the bottleneck is the lack of using technology in promoting. Usage of technology especially mobile based application in tourism sector allows both parties to connect and increases the accessibility of information. The final outcome of this research project is very beneficial for the tourists. This mobile application is much more convenient than using a booklet since the tourists can easily install the application and use it without much difficulty. It replaces the traditional tour guides by providing important services for the tourists. Finally, this mobile application enables tourists who come to visit Benishangul Gumuz region in Ethiopia be able to locate tourism sites without difficulty of finding information.

\section{Recommendation}

Since this mobile application is the first for the region's tourism, it has its own limitations because of the lack of infrastructure and mapping of the sites by google maps. The project team highly recommend that the regional state of Benishangul Gumuz have to focus on fulfilling the infrastructure and mapping of all tourism 
sites. Then the application can calculate both distance and time from the tourism sites.

\section{Acknowledgment}

First of all, we would like to thank almighty God for making this happen. Our second thanks goes to Benishangul Gumuz regional state tourism bureau for providing us data. The last but not the least thank is for Abebe Rorissa (PhD) for his contribution in publishing process.

\section{Authors Contributions}

Abeselom Befekadu Wolde: Proposing the idea, requirement analysis, collecting and analyzing the data, design and develop the prototype, writing the manuscript and submit for publication.

Yimer Amedie Muhie: Collect part of data and resources, reviewing and editing the manuscript.

\section{Ethics}

This article is original and contains unpublished material. The corresponding author confirms that all of the other authors have read and approved the manuscript and no ethical issues involved.

\section{References}

Al Thunibat, A., Mat Zin, N.A., \& Sahari, N. (2011). Mobile government user requirements model. Journal of E-Governance, 34, 104-111.

Citymapper. (2020, April 10). Retrieved from https://citymapper.com/istanbul?lang=en

Hevner, A. R., March, S. T., Park, J., \& Ram, S. (2004). Design science in information systems research. MIS quarterly, 75-105.

Oinas-Kukkonen, H., \& Kurkela, V. (2003, May). Developing successful mobile applications. In International Conference on Computer Science and Technology (IASTED) (pp. 50-54).

Sasol eBirds. (2020, April 10). Mobile Apps. https://www.sasolbirds.co.za/mobile-app.php

Splittr. (2020, April 10). Retrieved from https://www.splittr.io/

Tripit. (2020, April 10). An easier trip, every time. https://www.tripit.com/web 